Turkish Journal of Mathematics

http: / journals.tubitak.gov.tr/math/

Research Article

Turk J Math

(2021) 45: $288-299$

(C) TÜBİTAK

doi:10.3906/mat-2008-83

\title{
Modularly equidistant numerical semigroups
}

\author{
José Carlos ROSALES ${ }^{1}$, Manuel Baptista BRANCO ${ }^{2, *}$, , Márcio André TRAESEL $^{3}$ (D) \\ ${ }^{1}$ Department of Algebra, University of Granada, Granada, Spain \\ ${ }^{2}$ Department of Mathematics, University of Évora, Évora, Portugal \\ ${ }^{3}$ Department of Mathematics, Federal Institute of São Paulo, Caraguatatuba, Brazil
}

\begin{tabular}{llll} 
Received: 24.08 .2020 & Accepted/Published Online: 23.11.2020 $\quad$ Final Version: 21.01 .2021 \\
\hline
\end{tabular}

\begin{abstract}
If $S$ is a numerical semigroup and $s \in S$, we denote by next $\operatorname{dex}_{S}(s)=\min \{x \in S \mid s<x\}$. Let $a$ be an integer greater than or equal to two. A numerical semigroup is equidistant modulo $a$ if $\operatorname{next}_{S}(s)-s-1$ is a multiple of $a$ for every $s \in S$. In this note, we give algorithms for computing the whole set of equidistant numerical semigroups modulo $a$ with fixed multiplicity, genus, and Frobenius number. Moreover, we will study this kind of semigroups with maximal embedding dimension.
\end{abstract}

Key words: Embedding dimension, Frobenius number, genus, multiplicity, modularly equidistant numerical semigroups, MED semigroups, numerical semigroup

\section{Introduction}

Let $\mathbb{Z}$ be the set of integers an let $\mathbb{N}=n \in \mathbb{Z} \mid n \geqslant 0$ the set of nonnegative integers. A submonoid of $(\mathbb{N},+)$ is a subset of $\mathbb{N}$ closed addition and containing 0 . A submonoid of $(\mathbb{N},+)$ with finite complement in $\mathbb{N}$ is a numerical semigroup.

Given a numerical semigroup $S$ and $s \in S$, we denote by $\operatorname{next}_{S}(s)=\min \{x \in S \mid s<x\}$. For $a \in \mathbb{N} \backslash\{0,1\}$, we say that $S$ is an equidistant numerical semigroup modulo $a$ if $\operatorname{next}_{S}(s)-s-1$ is a multiple of $a$ for every $s \in S$.

We denote by

$$
\mathrm{E}(a)=\{S \mid S \text { is equidistant numerical semigroup modulo } a\} .
$$

Our aim in this note is to study of this kind of numerical semigroups.

This work is a generalization of the study of the parity numerical semigroups [3]. Indeed a numerical semigroup $S$ is parity if $s+\operatorname{next}_{S}(s)$ is odd for every $s \in S$. Clearly $s+\operatorname{next}_{S}(s)$ is odd if and only if $\operatorname{next}_{S}(s)-s-1$ is a multiple of 2 . Therefore, the parity numerical semigroups are equidistant numerical semigroups modulo 2 .

A numerical semigroup $S$ is prefect if $\{x-1, x+1\} \subseteq S$ then $x \in S$ (see for instance $[4,5]$ ). Observe that every equidistant modularly numerical semigroup is a perfect numerical semigroup. In fact, if $S$ is equidistant numerical semigroup modulo $a$ and $\{x-1, x+1\} \subseteq S$ then $x \in S$, because otherwise $\operatorname{next}_{S}(x-1)=x+1$ and then $\operatorname{next}_{S}(x-1)-(x-1)-1=x+1-x+1-1=1$ that is not a multiple of $a$.

*Correspondence: mbb@uevora.pt 2010 AMS Mathematics Subject Classification: 20M14, 11D07 
ROSALES et al./Turk J Math

We briefly outline the structure of this note. In Section 2 , we will order the elements of $\mathrm{E}(a)$ in a tree rooted in $\mathbb{N}$. We will characterize the sons of a vertex and this will allow us to build recursively the elements of $\mathrm{E}(a)$.

If $S$ is a numerical semigroup, then $\operatorname{m}(S)=\min (S \backslash\{0\}), \mathrm{F}(S)=\max \{z \in \mathbb{Z} \mid z \notin S\}$ and $\mathrm{g}(S)=$ $\operatorname{card}(\mathbb{N} \backslash S)$ (cardinality of $\mathbb{N} \backslash S$ ) are three important invariants of $S$ called multiplicity, Frobenius number, and genus of $S$, respectively. See for instance [6] and [2] to understand the importance of the study of these invariants.

As a consequence of the results of Section 2, we present an algorithmic procedure to compute all elements in $\mathrm{E}(a)$ with a given multiplicity and genus in Section 3. In Section 4, we get an algorithm for computing the set $\mathrm{E}(a)$ with given Frobenius number and we also characterize the maximal elements of this set.

An interesting class of numerical semigroups theory are the numerical semigroups with maximal embedding dimension (see [2]). In Section 5, we characterize the elements in $\mathrm{E}(a)$ with maximal embedding dimension.

\section{The tree associated to $\mathrm{E}(a)$}

In this section, we characterize the set $\mathrm{E}(a)$, for given $a$ integer greater than or equal to 2 . We see how this set can bee arranged in a tree.

Lemma 2.1 If $S \in \mathrm{E}(a)$ then $\mathrm{m}(S) \equiv 1(\bmod a)$.

Proof Clearly, $\operatorname{next}_{S}(0)=\mathrm{m}(S)$ and thus $\mathrm{m}(S)-0-1$ is a multiple of $a$. Therefore, we have that $\mathrm{m}(S) \equiv 1(\bmod a)$.

It is clear that $\mathbb{N}$ is a numerical semigroup that belongs to $\mathrm{E}(a)$. If $S \in \mathrm{E}(a)$ and $S \neq \mathbb{N}$ then $\mathrm{m}(S) \geq 2$, and so there exists $k \in \mathbb{N} \backslash\{0\}$ such that $\mathrm{m}(S)=1+k a$. Therefore, $a<\mathrm{m}(S)$.

Lemma 2.2 If $S \in \mathrm{E}(a) \backslash\{\mathbb{N}\}$, then $\{\mathrm{F}(S), \mathrm{F}(S)-1, \ldots, \mathrm{F}(S)-(a-1)\} \subseteq \mathbb{N} \backslash S$.

Proof Since $\mathrm{F}(S) \geq \mathrm{m}(S)-1 \geq a$ we have that $\{\mathrm{F}(S), \mathrm{F}(S)-1, \ldots, \mathrm{F}(S)-(a-1)\} \subseteq \mathbb{N} \backslash\{0\}$. Assume that there exists $i \in\{1, \ldots, a-1\}$ such that $\mathrm{F}(S)-i \in S$ and let $t=\min \{i \in\{1, \ldots, a-1\} \mid \mathrm{F}(S)-i \in S\}$. Then $\operatorname{next}_{S}(\mathrm{~F}(S)-t)=\mathrm{F}(S)+1$. As $S \in \mathrm{E}(a)$ then $\mathrm{F}(S)+1-(\mathrm{F}(S)-t)-1$ is a multiple of $a$. Hence, $t=l a$ for some $l \in \mathbb{N} \backslash\{0\}$ and consequently $t \geq a$, which is impossible.

Lemma 2.3 If $S \in \mathrm{E}(a) \backslash\{\mathbb{N}\}$, then $S \cup\{\mathrm{F}(S), \mathrm{F}(S)-1, \ldots, \mathrm{F}(S)-(a-1)\} \in \mathrm{E}(a)$.

Proof Since $a<\mathrm{m}(S)$, by applying Lemma 2.2, we get that $T=S \cup\{\mathrm{F}(S), \mathrm{F}(S)-1, \ldots, \mathrm{F}(S)-(a-1)\}$ is a numerical semigroup. Let $s \in S$ such that $\operatorname{next}_{S}(s)=\mathrm{F}(S)+1$. In order to conclude the proof, it suffices to show that $\operatorname{next}_{T}(s)-s-1$ is a multiple of $a$. As $\operatorname{next}_{T}(s)=\mathrm{F}(S)-(a-1)$ then $\operatorname{next}_{T}(s)-s-1$ is multiple of $a$ if and only if $\mathrm{F}(S)-(a-1)-s-1=\mathrm{F}(S)-s-a$ is multiple of $a$. However, this is true, because if $S \in \mathrm{E}(a)$ then $\mathrm{F}(S)+1-s-1=\operatorname{next}_{S}(s)-s-1$ is multiple of $a$.

The previous result can be viewed as a procedure to construct a sequence $\left\{S_{n} \mid n \in \mathbb{N}\right\}$ of elements in $\mathrm{E}(a)$. Given a numerical semigroup $S$, we define recursively

- $S_{0}=S$, 
ROSALES et al./Turk J Math

- $S_{n+1}= \begin{cases}S_{n} \cup\left\{\mathrm{F}\left(S_{n}\right), \mathrm{F}\left(S_{n}\right)-1, \ldots, \mathrm{F}\left(S_{n}\right)-(a-1)\right\} & \text { if } S_{n} \neq \mathbb{N} \\ \mathbb{N} & \text { otherwise. }\end{cases}$

The next result can be easily proved.

Proposition 2.4 If $S \in \mathrm{E}(a)$ and $\left\{S_{n} \mid n \in \mathbb{N}\right\}$ is the previous sequence, then there exists $k \in \mathbb{N}$ such that $S_{k}=\mathbb{N}$. Furthermore, for every $i \in\{0,1, \ldots, k-1\}$ then $\operatorname{card}\left(S_{i+1} \backslash S_{i}\right)=a$ and $k=\frac{\mathrm{g}(S)}{a}$.

We illustrate the previous result with an example.

Example 2.5 In the following sequence of elements of $\mathrm{E}(2)$, we have $k=3$.

$$
S_{0}=<5,6,7>\subsetneq S_{1}=<5,6,7,8,9>\subsetneq S_{2}=<3,4,5>\subsetneq S_{3}=\mathbb{N} .
$$

A graph $G$ is a pair $(V, E)$, where $V$ is a nonempty set and $E$ is a subset of $\{(v, w) \in V \times V \mid v \neq w\}$. The elements of $V$ and $E$ are called vertices and edges of $G$, respectively. A path of length $n$ connecting the vertices $u$ and $v$ of $G$ is a sequence of distinct edges of the form $\left(v_{0}, v_{1}\right),\left(v_{1}, v_{2}\right), \ldots,\left(v_{n-1}, v_{n}\right)$ with $v_{0}=u$ and $v_{n}=v$.

A graph $G$ is a tree if there exists a vertex $r$ (known as the root of $G$ ) such that for every other vertex $v$ of $G$, there exists a path connecting $v$ and $r$. If $(u, v)$ is a edge of the tree then we say that $u$ is a son of $v$.

We define the graph $G(\mathrm{E}(a))$ as graph whose vertices are the elements of $\mathrm{E}(a)$ and $(S, T) \in \mathrm{E}(a) \times \mathrm{E}(a)$ is an edge if $T=S \cup\{\mathrm{F}(S), \mathrm{F}(S)-1, \ldots, \mathrm{F}(S)-(a-1)\}$. From Proposition 2.4, we deduce the following.

Theorem 2.6 The graph $G(\mathrm{E}(a))$ is a tree with root equal to $\mathbb{N}$.

Note that the tree $G(\mathrm{E}(a))$ can be constructed recursively, from the root $\mathbb{N}$ in each step we are joining each of the vertices with its sons. Our next goal is to characterize the sons of an arbitrary vertex in the tree $G(\mathrm{E}(a))$. We distinguish two cases depending on whether or not the vertex is $\mathbb{N}$.

Lemma 2.7 The vertex $\mathbb{N}$ has a unique son in the tree $G(\mathrm{E}(a))$ that is $\{0, a+1, \rightarrow\}$.

Proof If $S$ is a son of $\mathbb{N}$, then $S \cup\{\mathrm{F}(S), \mathrm{F}(S)-1, \ldots, \mathrm{F}(S)-(a-1)\}=\mathbb{N}$ and thus $S=\{0, a+1, \rightarrow\}$.

If $\mathcal{X}$ is a nonempty subset of $\mathbb{N}$, then we will write $\langle\mathcal{X}\rangle$ for the submonoid of $(\mathbb{N},+)$ generated by $\mathcal{X}$, that is,

$$
\langle\mathcal{X}\rangle:=\left\{\sum_{i=1}^{n} \lambda_{i} x_{i} \mid n \in \mathbb{N} \backslash\{0\}, x_{1}, \ldots, x_{n} \in \mathcal{X}, \text { and } \lambda_{1}, \ldots, \lambda_{n} \in \mathbb{N}\right\} .
$$

It is well known (see for example [8]) that $\langle\mathcal{X}\rangle$ is a numerical semigroup if and only if $\operatorname{gcd}(\mathcal{X})=1$.

If $M$ is a submonoid of $(\mathbb{N},+)$ and $M=\langle\mathcal{X}\rangle$ then we say that $\mathcal{X}$ is a system of generators of $M$. Moreover, if $M \neq\langle\mathcal{Y}\rangle$ for all $\mathcal{Y} \varsubsetneqq \mathcal{X}$, then we say that $\mathcal{X}$ is a minimal system of generators of $S$. The following result can be deduced from [8, Corollary 2.8].

Corollary 2.8 If $M$ is a submonoid of $(\mathbb{N},+)$, then $M$ has a unique minimal system of generators, which in addition is finite. 
This minimal system of generators of $M$ will be denoted by $\operatorname{msg}(M)$.

Lemma 2.9 [7, Lemma 1.7] Let $S$ be a numerical semigroup and $x \in S$. Then $S \backslash\{x\}$ is a numerical semigroup if and only if $x \in \operatorname{msg}(S)$.

Proposition 2.10 Let $T \in \mathrm{E}(a) \backslash\{\mathbb{N}\}$. Then the set of sons of $T$, in the tree $G(\mathrm{E}(a))$, is equal to $\{T \backslash\{x, x+1, \ldots, x+(a-1)\} \mid\{x, x+1, \ldots, x+(a-1)\} \subseteq \operatorname{msg}(T)$ and $x>\mathrm{F}(T)\}$.

Proof If $T \in \mathrm{E}(a) \backslash\{\mathbb{N}\}$, then by Lemma 2.1, we have that $\mathrm{m}(T)>a$. Since $S$ is a son of $T$ then $T=S \cup\{\mathrm{F}(S), \mathrm{F}(S)-1, \ldots, \mathrm{F}(S)-(a-1)\}$. Hence, $\{\mathrm{F}(S), \mathrm{F}(S)-1, \ldots, \mathrm{F}(S)-(a-1)\} \subseteq \operatorname{msg}(T)$ and $\mathrm{F}(S)-(a-1)>\mathrm{F}(T)$.

Conversely, if $\{x, x+1, \ldots, x+(a-1)\} \subseteq \operatorname{msg}(T)$ and $x>\mathrm{F}(T)$ then, using repeatedly Lemma 2.9, we obtain that $S=T \backslash\{x, x+1, \ldots, x+(a-1)\}$ is a numerical semigroup with $\mathrm{F}(S)=x+a-1$. Therefore, $T=S \cup\{\mathrm{F}(S), \mathrm{F}(S)-1, \ldots, \mathrm{F}(S)-(a-1)\}$ and thus $S$ is a son of $T$.

Example 2.11 Let us construct recursively the tree $G(\mathrm{E}(2))$.

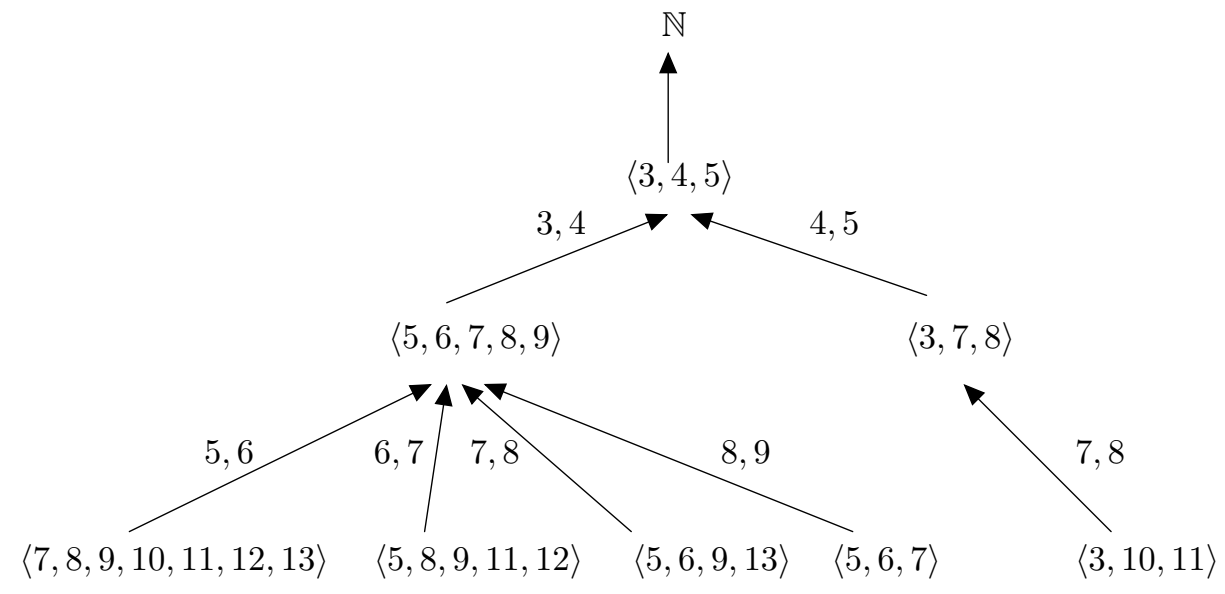

The numbers that appear on either side of the edges is the elements that we remove from the semigroup to obtain its son. For example $\langle 3,4,5\rangle \backslash\{3,4\}=\langle 5,6,7,8,9\rangle$.

Observe that $\mathrm{E}(a)$ has infinite cardinality, because $\{0, k a+1, \rightarrow)\} \in \mathrm{E}(a)$ for all $k \in \mathbb{N}$.

\section{The set $\mathrm{E}(a)$ with a given multiplicity and genus}

We will denote by $\mathrm{E}(a, m)=\{S \in \mathrm{E}(a) \mid \mathrm{m}(S)=m\}$. By Lemma 2.1, we obtain that $\mathrm{E}(a, m) \neq \emptyset$ if and only if $m=k a+1$ for some $k \in \mathbb{N}$. It is clear that $\mathrm{E}(a, 1)=\{\mathbb{N}\}$. 
ROSALES et al./Turk J Math

From now on, assume that $m=k a+1$ with $k \in \mathbb{N} \backslash\{0\}$. It is clear that maximum element in $\mathrm{E}(a)$ is $\langle m, m+1, \ldots, 2 m-1\rangle=\{0, m, \rightarrow\}$. From Lemma 2.3, we deduce the next result.

Lemma 3.1 If $S \in \mathrm{E}(a, m)$ and $S \neq\{0, m, \rightarrow\}$, then $S \cup\{\mathrm{F}(S), \mathrm{F}(S)-1, \ldots, \mathrm{F}(S)-(a-1)\} \in \mathrm{E}(a, m)$.

The previous lemma allows us to define recurrently the sequence $\left\{S_{n} \mid n \in \mathbb{N}\right\}$ of elements of $S \in \mathrm{E}(a, m)$. If $S \in \mathrm{E}(a, m)$, then

- $S_{0}=S$,

- $S_{n+1}= \begin{cases}S_{n} \cup\left\{\mathrm{F}\left(S_{n}\right), \mathrm{F}\left(S_{n}\right)-1, \ldots, \mathrm{F}\left(S_{n}\right)-(a-1)\right\} & \text { if } S_{n} \neq\{0, m, \rightarrow\} \\ \{0, m, \rightarrow\} & \text { otherwise. }\end{cases}$

The next result has immediate proof.

Proposition 3.2 If $S \in \mathrm{E}(a, m)$ and $\left\{S_{n} \mid n \in \mathbb{N}\right\}$ is the previous sequence, then there exists $k \in \mathbb{N}$ such that $S_{k}=\{0, m, \rightarrow\}$. Moreover, for every $i \in\{0,1, \ldots, k-1\}$ then $\operatorname{card}\left(S_{i+1} \backslash S_{i}\right)=a$ and $k=\frac{\mathrm{g}(S)-(m-1)}{a}$.

As a consequence of Lemma 2.1 and Proposition 3.2, we have the following result.

Corollary 3.3 If $S \in \mathrm{E}(a, m)$ then $\mathrm{g}(S)$ is a multiple of $a$.

We define the graph $G(\mathrm{E}(a, m))$ as follows: $\mathrm{E}(a, m)$ is its set of vertices and $(S, T) \in \mathrm{E}(a) \times \mathrm{E}(a)$ is an edge if $T=S \cup\{\mathrm{F}(S), \mathrm{F}(S)-1, \ldots, \mathrm{F}(S)-(a-1)\}$.

The following result is a consequence of Propositions 2.10 and 3.2 .

Theorem 3.4 The graph $G(\mathrm{E}(a, m))$ is a tree rooted in $\{0, m, \rightarrow\}$. Moreover, the set of sons of $T$ is equal to

$$
\{T \backslash\{x, x+1, \ldots, x+(a-1)\} \mid\{x, x+1, \ldots, x+(a-1)\} \subseteq \operatorname{msg}(T), x>\mathrm{F}(T)) \text { and } x \neq m\}
$$

Example 3.5 We are going to build the tree $G(\mathrm{E}(2,5))$. 


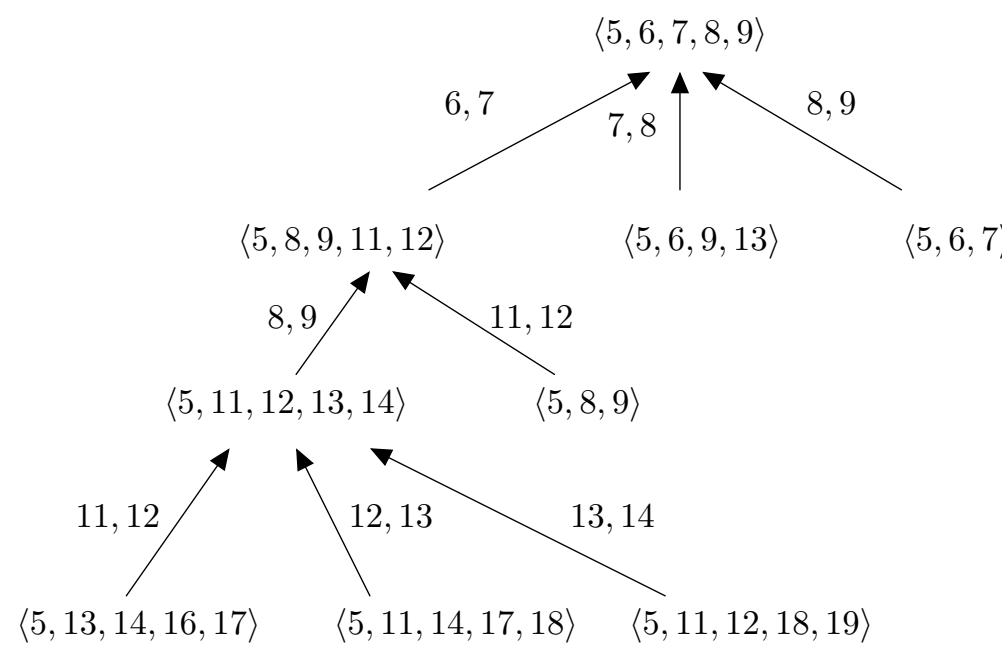

Observe that for $t \in \mathbb{N} \backslash\{0\}$ we have that $\langle m\rangle \cup\{t m, \rightarrow\} \in \mathrm{E}(a, m)$ and so $\mathrm{E}(a, m)$ has infinite cardinality. Our next aim in this section will be to show an algorithm that allows us to compute the set $\mathrm{E}(a, m)$ with given genus.

If $G=(V, E)$ is a tree and $v \in V$, then the depth of $v$, which we will denote by $d(v)$, is the length of a unique path that connects $v$ with the root. Given $k$ a nonnegative integer, denote by $N(G, k)=$ $\{v \in V \mid d(v)=k\}$.

As a consequence of Proposition 3.2 we have the following result.

Proposition 3.6 Let $S \in \mathrm{E}(a, m)$. Then $S \in N(G(\mathrm{E}(a, m)), k)$ if and only if $\mathrm{g}(S)=m-1+a k$.

The next lemma follows immediately from the definitions.

Lemma 3.7 If $k \in \mathbb{N}$, then $N(G(\mathrm{E}(a, m)), k+1)=\{S \mid S$ is a son of an element in $N(G(\mathrm{E}(a, m)), k)\}$.

We are already able to give an algorithm that allows us to compute the set $\mathrm{E}(a, m)$ with a given genus. Note that $S \in \mathrm{E}(a, m)$ then $\{1, \ldots, m-1\} \subseteq \mathbb{N} \backslash S$ and thus $\mathrm{g}(S) \geq m-1$. Moreover, by Corollary 3.3, we have that $\mathrm{g}(S)$ is a multiple of $a$. 


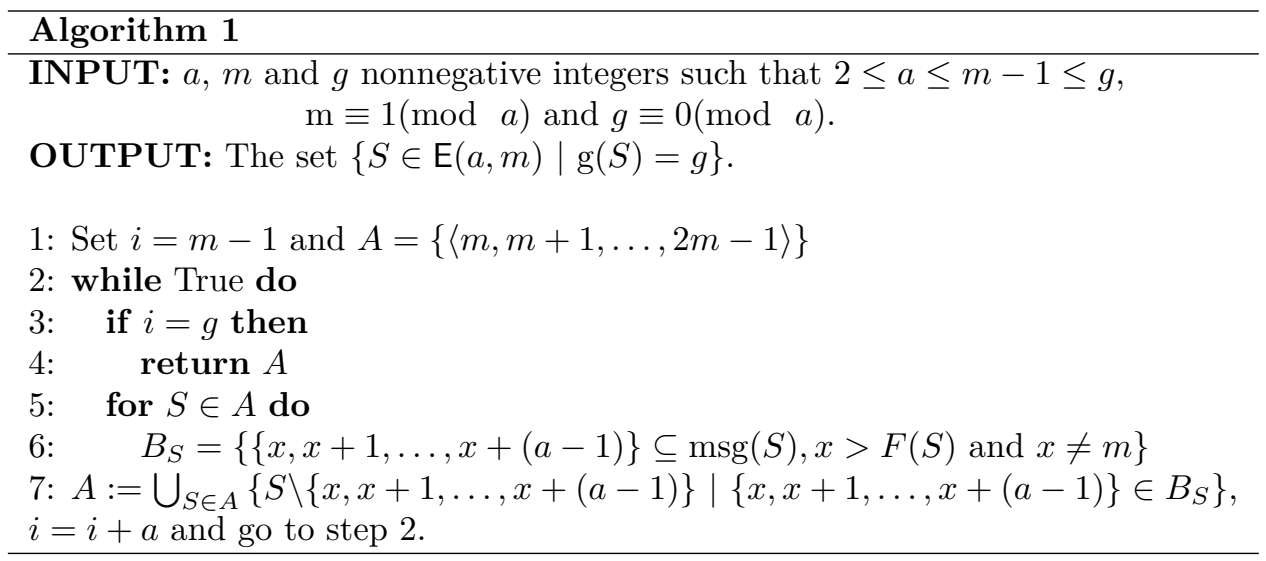

Example 3.8 Let us compute the set $\{S \in \mathrm{E}(2,5) \mid \mathrm{g}(S)=8\}$ using Algorithm 1 .

1. Set $i=4$ and $A=\{\langle 5,6,7,8,9\rangle\}$.

2. The first loop constructs $B_{\langle 5,6,7,8,9\rangle}=\{\{6,7\},\{7,8\},\{8,9\}\}$ then $A=\{\langle 5,6,7,8,9\rangle \backslash\{6,7\},\langle 5,6,7,8,9\rangle \backslash\{7,8\},\langle 5,6,7,8,9\rangle \backslash\{8,9\}\}, i=6$,

3. the second loop constructs $B_{\langle 5,8,9,11,12\rangle}=\{\{8,9\},\{11,12\}\}, B_{\langle 5,6,9,13\rangle}=\emptyset$ and $B_{\langle 5,6,7\rangle}=\emptyset$ then $A=\{\langle 5,8,9,11,12\rangle \backslash\{8,9\},\langle 5,8,9,11,12\rangle \backslash\{11,12\}\}, i=8$.

Hence, $\{S \in \mathrm{E}(2,5) \mid \mathrm{g}(S)=8\}=\{\langle 5,11,12,13,14\rangle,\langle 5,8,9\rangle\}$.

\section{The set $\mathrm{E}(a)$ with a given Frobenius number}

Our aim in this section will be to show an algorithm that allows us to compute the set $\mathrm{E}(a)$ with a given Frobenius number. If $S$ is a numerical semigroup such that $S \neq \mathbb{N}$, then we have that $\mathrm{F}(S) \geq \mathrm{m}(S)-1$. Besides, by Lemma 2.1, if $S \in \mathrm{E}(a) \backslash\{\mathbb{N}\}$ then $\mathrm{m}(S)=1+k a$ for some $k \in \mathbb{N} \backslash\{0\}$. Since $\mathrm{m}(S) \leq \mathrm{F}(S)+1$ and $\mathrm{m}(S)=1+k a$ then $k \leq \frac{\mathrm{F}(S)}{a}$.

Given a rational number $q$ we denote by $\lfloor q\rfloor$ its integer part, that is, $\lfloor q\rfloor=\max \{z \in \mathbb{Z} \mid z \leq q\}$. We can announce the following result.

Lemma 4.1 Let $\mathrm{F}(S)$ be an integer greater than or equal to two. Then

$\{S \in \mathrm{E}(a) \mid \mathrm{F}(S)=F\}=\bigcup_{k=1}^{\left\lfloor\frac{F}{a}\right\rfloor}\{S \in \mathrm{E}(a, k a+1) \mid \mathrm{F}(S)=F\}$.

Clearly, $\{S \in \mathrm{E}(a, k a+1) \mid \mathrm{F}(S)=k a\}=\{0, k a+1 \rightarrow\}$.

Lemma 4.2 Let $k \in \mathbb{N} \backslash\{0\}$ such that $k a+1<F$. Then $\{S \in \mathrm{E}(a, k a+1) \mid \mathrm{F}(S)=F\} \neq \emptyset$ if and only if $F \bmod (k a+1) \notin\{0,1, \ldots, a-1\}$.

Proof Necessity. If $S \in \mathrm{E}(a, k a+1)$ and $\mathrm{F}(S)=F$ then, applying Lemma 2.2, we have that $\{\mathrm{F}(S), \mathrm{F}(S)-1, \ldots, \mathrm{F}(S)-(a-1)\} \subseteq \mathbb{N} \backslash S$. Therefore, $\{\mathrm{F}(S), \mathrm{F}(S)-1, \ldots, \mathrm{F}(S)-(a-1)\} \cap\langle k a+1\rangle=\emptyset$ and so $F \bmod (k a+1) \notin\{0,1, \ldots, a-1\}$. 
Sufficiency. Assume that $r=F \bmod (k a+1)$. Then $F=q(k a+1)+r$ for some $q \in \mathbb{N} \backslash\{0\}$ and $r \in\{a, \ldots, k a\}$. Hence we obtain that

$$
S=\{0, k a+1,2(k a+1), \ldots, q(k a+1), q(k a+1)+1, \ldots, q(k a+1)+r-a, F+1, \rightarrow\} \in \mathrm{E}(a)
$$

and $\mathrm{F}(S)=F$.

Now by using Lemmas 4.1 and 4.2, in order to compute the set $\mathrm{E}(a)$ with a given Frobenius number $F$, it is enough to give an algorithm that computes this set with $m$ a positive integer and verifies that $2 \leq a \leq m-1$, $m<F, m \equiv 1(\bmod a)$, and $(F \bmod m) \notin\{0,1, \ldots, a-1\}$.

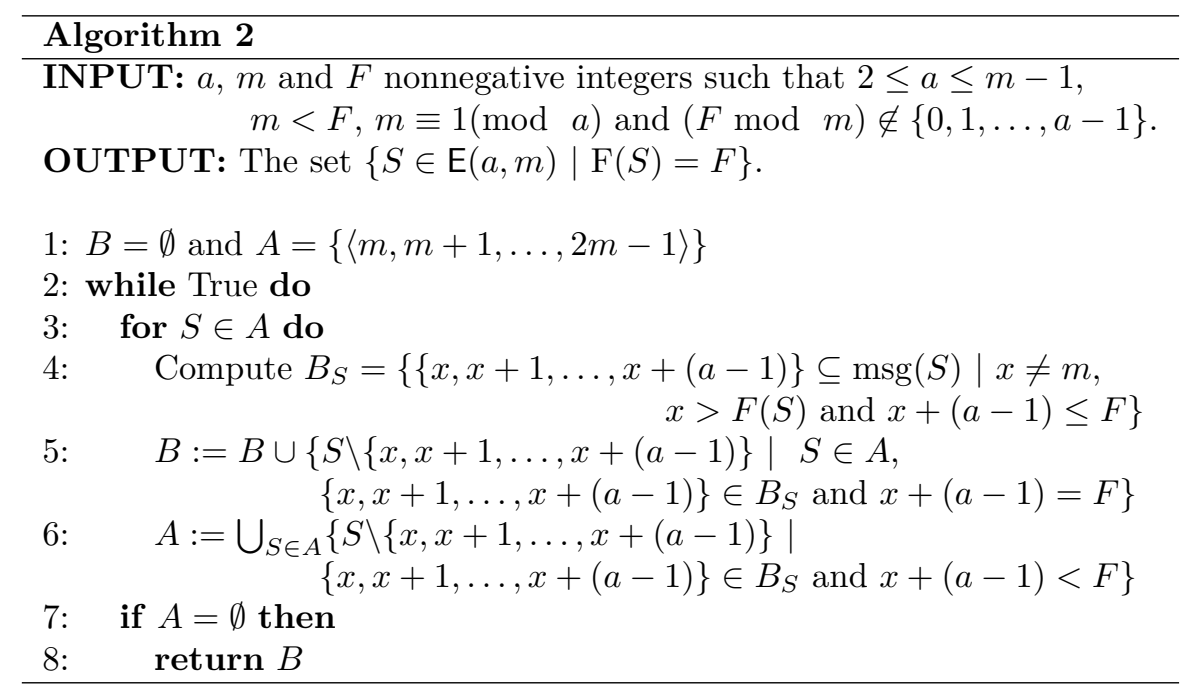

Next we illustrate this method with an example.

Example 4.3 Let us compute the set $\{S \in \mathrm{E}(2) \mid \mathrm{F}(S)=12\}$. First, by using Lemma 4.1, we have that $\{S \in \mathrm{E}(2) \mid \mathrm{F}(S)=12\}=\bigcup_{m \in\{3,5,7,9,11,13\}}\{S \in \mathrm{E}(2, m) \mid \mathrm{F}(S)=12\}$. From Lemma 4.2, we obtain that $\{S \in \mathrm{E}(2,3) \mid \mathrm{F}(S)=12\}=\emptyset$ and $\{S \in \mathrm{E}(2,11) \mid \mathrm{F}(S)=12\}=\emptyset$. Moreover, by the observation made after the Lemma 4.1, we get that $\{S \in \mathrm{E}(2,13) \mid \mathrm{F}(S)=12\}=\{0,13, \rightarrow\}$. Therefore, by using Algorithm 2, we have to compute the set $\{S \in \mathrm{E}(2, m) \mid \mathrm{F}(S)=12\}$ with $m \in\{5,7,9\}$.

For example, we will calculate the set $\{S \in \mathrm{E}(2,5) \mid \mathrm{F}(S)=12\}$.

1. Start $B=\emptyset$ and $A=\{\langle 5,6,7,8,9\rangle\}$.

2. The first loop constructs $B_{\langle 5,6,7,8,9\rangle}=\{\{6,7\},\{7,8\},\{8,9\}\}$

then $B=\emptyset$,

3. next constructs $A=\{\langle 5,6,7,8,9\rangle \backslash\{6,7\},\langle 5,6,7,8,9\rangle \backslash\{7,8\},\langle 5,6,7,8,9\rangle \backslash\{8,9\}\}$,

4. the second loop constructs $B_{\langle 5,8,9,11,12\rangle}=\{\{8,9\},\{11,12\}\}, B_{\langle 5,6,9,13\rangle}=\emptyset$ and $B_{\langle 5,6,7\rangle}=\emptyset$

then $B=\{\langle 5,8,9,11,12\rangle \backslash\{11,12\}\}$,

5. next constructs $A=\{\langle 5,8,9,11,12\rangle \backslash\{8,9\}\}$, 
6. the third loop constructs $B_{\langle 5,11,12,13,14\rangle}=\{\{11,12\}\}$

then $B=\{\langle 5,8,9\rangle,\langle 5,11,12,13,14\rangle \backslash\{11,12\}\}$,

7. next constructs $A=\emptyset$,

8. $\{S \in \mathrm{E}(2,5) \mid \mathrm{F}(S)=12\}=\{\langle 5,8,9\rangle,\langle 5,13,14,16,17\rangle\}$.

Next we are interested in characterizing of the maximal elements in the set $\{S \in \mathrm{E}(a) \mid \mathrm{F}(S)=F\}$. The next result is well known.

Lemma 4.4 [9, Lemma 10] Let $S$ and $T$ be two numerical semigroups such that $S \subsetneq T$ and $x=\max (T \backslash S)$. Then $S \cup\{x\}$ is a numerical semigroup.

Proposition 4.5 Let $\{S, T\} \subseteq \mathrm{E}(a)$ such that $S \subsetneq T, x=\max (T \backslash S)$ and let $s \in S$ such that $s<x<$ $\operatorname{next}_{S}(s)$. If $\left\{x_{1}<x_{2}<\cdots<x_{r}\right\}=\left\{t \in T \mid s<t<\operatorname{next}_{S}(s)\right\}$ then $S \cup\left\{x_{1}, x_{2}, \ldots, x_{r}\right\} \in \mathrm{E}(a), r$ is a multiple of $a$ and $S \cup\left\{x_{r}, x_{r-1}, \ldots, x_{r-(a-1)}\right\} \in \mathrm{E}(a)$.

Proof By repeatedly applying Lemma 4.4, we get that $S \cup\left\{x_{1}, x_{2}, \ldots, x_{r}\right\}$ is a numerical semigroup. Moreover, as $\{S, T\} \subseteq \mathrm{E}(a)$ such that $S \subseteq T$, we deduce that $S \cup\left\{x_{1}, x_{2}, \ldots, x_{r}\right\} \in \mathrm{E}(a)$.

Since $s<x_{1}<\cdots<x_{r}<\operatorname{next}_{S}(s)$ are consecutive elements of $T$ and $T \in \mathrm{E}(a)$, then there exist $\left\{k_{1}, \ldots k_{r+1}\right\} \subseteq \mathbb{N}$ such that $x_{1}=s+k_{1} a+1, x_{2}=s+k_{1} a+1+k_{2} a+1, \ldots, x_{r}=s+k_{1} a+1+\cdots+k_{r} a+1$ and thus $\operatorname{next}_{S}(s)=s+k_{1} a+1+\cdots+k_{r} a+1+k_{r+1} a+1$. Therefore, next $\operatorname{ne}_{S}(s)-s=k_{1} a+1+\cdots+k_{r} a+1+k_{r+1} a+1$. As $S \in \mathrm{E}(a)$ then $\operatorname{next}_{S}(s)-s-1=t a$ for some $t \in \mathbb{N}$. Consequently, $k_{1} a+1+\cdots+k_{r} a+1+k_{r+1} a+1=t a+1$. Then $\left(k_{1}+\cdots+k_{r+1}\right) a+r+1=t a+1$ and thus $r$ is a multiple of $a$. Assume that $r=l a$ for some $l \in \mathbb{N} \backslash\{0\}$

To conclude the proof, we check that $S \cup\left\{x_{(l-1) a+1}, x_{(l-1) a+2}, \ldots, x_{(l-1) a+a}\right\} \in \mathrm{E}(a)$. In order to see this, it is enough to see that $x_{(l-1) a+1}-s-1$ is a multiple of $a$. This is true because $x_{(l-1) a+1}-s-1=$ $s+k_{1} a+1+k_{2} a+1+\cdots+k_{(l-1) a+1} a+1-s-1=\left(k_{1}+\cdots+k_{(l-1)+1}\right) a+(l-1) a+1-1$ is a multiple of $a$.

Given a sequence of nonnegative integers $n_{1}<n_{2}<\cdots<n_{p}$, we say that it is equidistant modulo $a$ if $n_{i+1}-n_{i}-1$ is a multiple of $a$ for all $i \in\{1, \ldots, p-1\}$.

Let $S$ be a numerical semigroup. An element of $s \in S$ is called a-refinable if there exists $\left\{x_{1}<x_{2}<\cdots<x_{a}\right\} \subseteq\left\{x \in \mathbb{N} \mid s<x<\operatorname{next}_{S}(s)\right.$ and $\left.x_{a}<\mathrm{F}(S)\right\}$ such that $S \cup\left\{x_{1}, x_{2}, \ldots, x_{a}\right\}$ is a numerical semigroup and the sequence $s, x_{1}, x_{2}, \ldots, x_{a}, \operatorname{next}_{S}(s)$ is equidistant modulo $a$. We denote by $\mathcal{R}(S)=$ $\{s \in S \mid S$ is a-refinable $\}$.

Theorem 4.6 Let $S \in \mathrm{E}(a)$ with $\mathrm{F}(S)=F$. Then $S$ is a maximal element in the set $\{T \in \mathrm{E}(a) \mid \mathrm{F}(T)=F\}$ if and only if $\mathcal{R}(S)=\emptyset$.

Proof Necessity. If $\mathcal{R}(S) \neq \emptyset$, then there exists $s \in \mathcal{R}(S)$. Hence, there exist $\left\{x_{1}<x_{2}<\cdots<x_{a}\right\} \subseteq$ $\mathbb{N} \backslash\{0\}$ such that the sequence $s<x_{1}<x_{2}<\cdots<x_{a}<\operatorname{next}_{S}(s)$ is equidistant modulo $a$ with $x_{a}<$ $F$ and $S \cup\left\{x_{1}, x_{2}, \ldots, x_{a}\right\}$ is a numerical semigroup. We deduce that $S \cup\left\{x_{1}, x_{2}, \ldots, x_{a}\right\} \in \mathrm{E}(a)$ with $\mathrm{F}\left(S \cup\left\{x_{1}, x_{2}, \ldots, x_{a}\right\}\right)=F$ contradicting the maximality of $S$. 
ROSALES et al./Turk J Math

Sufficiency. If we suppose that $S$ is not maximal, then there exists $T \in \mathrm{E}(a)$ with $\mathrm{F}(T)=F$ and $S \subsetneq T$. Let $x=\max (T \backslash S)$ and $s \in S$ such that $s<x<\operatorname{next}_{S}(s)$. By applying Proposition 4.5, we obtain that $s \in \mathcal{R}(S)$ and thus $\mathcal{R}(S) \neq \emptyset$.

\section{The elements of $\mathrm{E}(a)$ with maximal embedding dimension}

Let $S$ be a numerical semigroup. The cardinality of $\operatorname{msg}(S)$ is known as the embedding dimension of $S$ and it is denoted here by e $(S)$. In [8, Proposition 2.10] shows that $\mathrm{e}(S) \leq \mathrm{m}(S)$. We say that $S$ has maximal embedding dimension (MED-semigroup) if e $(S)=m(S)$. The following result can be deduced from [2, Proposition I.2.9].

Lemma 5.1 Let $S$ be a numerical semigroup. Then $S$ is a MED-semigroup if and only if $\{s-\mathrm{m}(S) \mid s \in S \backslash\{0\}\}$ is a numerical semigroup.

Proposition 5.2 Let $S$ be a MED-semigroup. Then $S \in \mathrm{E}(a)$ if and only if $T=\{s-\mathrm{m}(S) \mid s \in S \backslash\{0\}\}$ is an element of $\mathrm{E}(a)$ and $\mathrm{m}(S) \equiv 1(\bmod a)$.

Proof Necessity. By Lemmas 5.1 and 2.1, we have that $T$ is a numerical semigroup and $\mathrm{m}(S) \equiv 1(\bmod a)$, respectively. To conclude the proof, it suffices to see that $\operatorname{next}_{T}(t)-t-1$ is a multiple of $a$ for every $t \in T$. If $t \in T$, then there exists $s \in S \backslash\{0\}$ such that $t=s-\mathrm{m}(S)$ and so $\operatorname{next}_{T}(t)=\operatorname{next}_{S}(t)-\mathrm{m}(S)$. Therefore, $\operatorname{next}_{T}(t)-t-1=\operatorname{next}_{S}(s)-\mathrm{m}(S)-(s-\mathrm{m}(S))-1=\operatorname{next}_{S}(s)-s-1$ is a multiple of $a$, because $S \in \mathrm{E}(a)$.

Sufficiency. Let us see that $S \in \mathrm{E}(a)$, that is, if $s \in S$ then $\operatorname{next}_{S}(s)-s-1$ is a multiple of $a$. If $s=0$, then $\operatorname{next}_{S}(s)-0-1=\mathrm{m}(S)-1$ is a multiple of $a$. If $s \neq 0$, then $s-m(s)=t \in T$ and $\operatorname{next}_{T}(t)=\operatorname{next}_{S}(s)-\mathrm{m}(S)$. Hence, $\operatorname{next}_{S}(s)-s-1=\operatorname{next}_{T}(t)-t-1$ is multiple of $a$, because $T \in \mathrm{E}(a)$.

From Lemma 5.1, it is easy to deduce the following result.

Lemma 5.3 Let $S$ be a numerical semigroup and $x \in S \backslash\{0\}$. Then $S(x)=(\{x\}+S) \cup\{0\}$ is a MED-semigroup with multiplicity x. Moreover, every MED-semigroup is of this form.

Proposition 5.4 Let $S \in \mathrm{E}(a)$ and $x \in S \backslash\{0\}$ such that $x \equiv 1(\bmod a)$. Then $S(x)=(\{x\}+S) \cup\{0\}$ is an equidistant MED-semigroup modulo a. Moreover, every equidistant MED-semigroup modulo a is of this form.

Proof By Lemma 5.3, we obtain that $S(x)$ is a MED-semmigroup with multiplicity $x$. Clearly $S=$ $\{s-x \mid s \in S(x) \backslash\{0\}\}$ and thus, applying Proposition 5.2, we obtain that $S(x)$ is equidistant modulo $a$.

Let $T$ be an equidistant MED-semigroup modulo $a$. Then by Proposition 5.2, we get that $S=$ $\{T-\mathrm{m}(T) \mid t \in T \backslash\{0\}\} \in \mathrm{E}(a)$ and $\mathrm{m}(T) \equiv 1(\bmod a)$. Therefore, $T=(\{\mathrm{m}(T)\}+S) \cup\{0\}$ with $S \in \mathrm{E}(a)$ and $\mathrm{m}(T) \in S$ such that $\mathrm{m}(T) \equiv 1(\bmod a)$.

Let $S$ be a numerical semigroup and let $n \in S \backslash\{0\}$. The Apéry set (named so in honour of [1]) of $n$ in $S$ is

$$
\operatorname{Ap}(S, n):=\{s \in S \mid s-n \notin S\}
$$

Lemma 5.5 [8, Lemma 2.4] Le $S$ be a numerical semigroup and $n \in S \backslash\{0\}$. Then $\operatorname{Ap}(S, n)=\{0=$ $w(0), w(1), \ldots, w(n-1)\}$, where $w(i)$ is the least element in $S$ congruent with $i$ modulo $n$, for all $i \in$ $\{0, \ldots, n-1\}$. 
ROSALES et al./Turk J Math

Observe that the above lemma in particular implies that the cardinality of $\operatorname{Ap}(S, n)$ is $n$. From [10], we can deduce the next result.

Proposition 5.6 Le $S$ be a numerical semigroup, $n \in S \backslash\{0\}$ and $T=(\{n\}+S) \cup\{0\}$. Then the following conditions hold:

1. $T$ is MED-semigroup

2. $\mathrm{m}(T)=n$.

3. $\mathrm{F}(T)=\mathrm{F}(S)+n$.

4. $\mathrm{g}(T)=\mathrm{g}(S)+n-1$.

5. $\operatorname{msg}(T)=\operatorname{Ap}(S, n)+\{n\}$.

Example 5.7 It is clear that $S=\langle 5,8,9\rangle$ is an an equidistant numerical semigroup modulo 2 . We have that 9 is an element in $S$ such that $9 \equiv 1(\bmod 2)$. Then from Proposition 5.4 , we obtain that $T=$ $(\{9\}+S) \cup\{0\}$ is an equidistant MED-semigroup modulo 2. Since $\mathrm{F}(S)=12, g(S)=8$ and $\mathrm{Ap}(S, 9)=$ $\{0,5,8,10,13,15,16,20,21\}$, by Proposition 5.6 we have that $\mathrm{F}(T)=12+9=21, \mathrm{~g}(T)=8+9-1=16$ and $\operatorname{msg}(T)=\{9,14,17,19,22,24,25,29,30\}$.

\section{Acknowledgments}

We would like to thank the referees for their comments and suggestions on the manuscript.

The first author was partially supported by MTM-2017-84890-P and by Junta de Andalucia group FQM-343. The second author is supported by the project FCT PTDC/MAT/73544/2006).

\section{References}

[1] Apéry R. Sur les branches superlinéaires de courbes algébriques. Comptes Rendus de l'Académie des Sciences 1946; Paris 222: 1198-2000 (in French).

[2] Barucci V, Dobbs DE, Fontana M. Maximality properties in numerical semigroups and applications to onedimensional analytically irreducible local domains. Memoirs of the American Mathematical Society 1997; 598: 95.

[3] Moreno-Frias MA, Rosales JC. Parity numerical semigroups. Hacettepe Journal of Mathematics and Statistics 2020; 49 (3): 1067-1075. doi: 10.15672/hujms.548289

[4] Moreno-Frias MA, Rosales JC. Perfect numerical semigroups. Turkish Journal of Mathematics 2019; 43: $1742-1754$. doi: 10.3906/mat-1901-111

[5] Moreno-Frias MA, Rosales JC. Perfect numerical semigroups with embedding dimension tree. Publicationes Mathematicae Debrecen 2020; 97/1-2: 77-84. doi: 10.5486/PMD.2020.8699

[6] Ramirez Alfonsín JL. The Diophantine Frobenius Problem. London, UK: Oxford University Press, 2005. doi: 10.1093/acprof:oso/9780198568209.001.0001

[7] Rosales JC. Numerical semigroups that differ from a symmetric numerical semigroups in one element. Algebra Colloquium 2008; 15 (01): 23-32. doi: 10.1142/S1005386708000035

[8] Rosales JC, García-Sánchez PA. Numerical semigroups. Developments in Mathematics. 20. New York, NY, USA: Springer 2009. doi: 10.1007/978-1-4419-0160-6 
ROSALES et al./Turk J Math

[9] Rosales JC, García-Sánchez PA, García-García JI, Jimenez-Madrid JA. The oversemigroups of a numerical semigroup. Semigroup Forum 2003; 67: 145-158. doi: 10.1007/s00233-002-0007-3

[10] Rosales JC. Principal ideals of a numerical semigrops. Bulletin of the Belgian Mathematical Society - Simon Stevin 2003; 10 (3): 329-343. 